\title{
An unbiased search for the signatures of protostars in the $\rho$ Ophiuchi A molecular cloud $\star, \star \star$
}

\section{Near-infrared observations}

\author{
T. Khanzadyan ${ }^{1}$, R. Gredel ${ }^{1}$, M. D. Smith ${ }^{2}$, and T. Stanke \\ 1 Max-Planck Institut für Astronomie, Königstuhl 17, 69117 Heidelberg, Germany \\ e-mail: khtig@mpia-hd.mpg.de; gredel@caha.es \\ 2 Armagh Observatory, Armagh BT61 9DG, Northern Ireland, UK \\ e-mail: mds@arm.ac.uk \\ ${ }^{3}$ MPI für Radioastronomie, Auf dem Hügel 69, 53121, Bonn, Germany \\ e-mail: tstanke@mpifr-bonn.mpg.de
}

Received 6 May 2004 / Accepted 22 June 2004

\begin{abstract}
We present an unbiased search for molecular hydrogen emission in the L1688 cloud within the $\rho$ Ophiuchi molecular cloud complex. Our near-infrared survey covers a connected region of extent $35^{\prime} \times 35^{\prime}$. We detect several new $\mathrm{H}_{2}$ flows but the total number of detected outflows is low and is consistent with the paucity of Class 0 and Class 1 sources in the molecular cloud. From the spatial distribution, their collimation and the individual shapes of the bow shocks, we suggest possible candidates for the outflow sources. Most of the candidate driving sources are deeply embedded in dense cores of the molecular cloud. A very young outflow arises from the newly discovered Class 0 source MMS 126. Two major outflows in the NE-SW direction arise from the YLW 15 and YLW 16 Class I sources. Three additional outflows, which both extend over several arcminutes, arise from the Class I sources YLW 31 and YLW 52. Flow directions are generally NE-SW, perpendicular to the elongation directions of the cloud filaments. The apparent extents of molecular flows are related to either the widths of cloud filaments or to the separation between filaments. The estimated jet power needed to continuously drive and excite the detected portions of the shocked $\mathrm{H}_{2}$ outflows lies in the range $0.02-0.2 L_{\odot}$. Given the critical dependence on the environment, however, the total sizes and powers of the outflows may be considerably larger.
\end{abstract}

Key words. stars: formation - ISM: jets and outflows - ISM: clouds

\section{Introduction}

The $\rho$ Ophiuchi molecular cloud complex is one of the nearest active star forming regions (see Reipurth et al. 1991; Lada et al. 1993, for reviews). The cloud complex contains a number of distinct dark clouds (Lynds 1962) with a total mass estimated to be $10^{4} M_{\odot}$ (de Geus et al. 1990). The filamentary and clumpy distribution of the molecular gas has been revealed through large-scale CO surveys (de Geus et al. 1990; Loren 1989a,b). Embedded in the filaments are dense clumps, recognised by mapping in emission lines of $\mathrm{NH}_{3}, \mathrm{DCO}^{+}$and $\mathrm{C}^{18} \mathrm{O}$, with properties similar to the cores in Taurus (Myers \& Benson 1983; Benson \& Myers 1989; Loren et al. 1990; Tachihara et al. 2000). Typically, clump masses are in the range 10-30 $\mathrm{M}_{\odot}$.

* Based on observations collected at the European Southern Observatory, Chile (ESO No. 67.C-0284), and the German-Spanish Astronomical Center, Calar Alto, operated jointly by Max-Planck Institut für Astronomie and Instituto de Astrofisica de Andalucia.

$\star \star$ Table 2 is only available in electronic form at the CDS via anonymous ftp to cdsarc.u-strasbg.fr $(130.79 .128 .5)$ or via http://cdsweb.u-strasbg.fr/cgi-bin/qcat?J/A+A/426/171
In turn, these clumps contain fragments termed cores of mass $0.10 M_{\odot}-10 M_{\odot}$, some of which already contain protostars and young stars (Motte et al. 1998; Johnstone et al. 2000; Stanke et al. 2004).

It is believed that star formation has been triggered by ionisation fronts and winds from the Upper Scorpius-Centaurus OB association which is located to the west of the $\rho$ Oph region (e.g., Loren 1989a; de Geus et al. 1990). The most active part of the $\rho$ Oph molecular cloud complex is L1688. It harbours a remarkably dense and compact population of young stellar objects with $>50$ stars $\mathrm{pc}^{-2}$ at different evolutionary stages. The vast majority of these are relatively old $\mathrm{T}$ Tauri stars (Class I and II) although there are a few so-called protostars (Class 0 and I) amongst them (Wilking et al. 1989; Barsony et al. 1997; Bontemps et al. 2001). The population of young stars has been studied across the spectrum: in the radio (Andre et al. 1987; Leous et al. 1991; Mezger et al. 1992; Bontemps et al. 1996), the millimetre (Andre et al. 1990), the infrared (e.g., Barsony et al. 1997; Luhman \& Rieke 1999; Bontemps et al. 2001; Allen et al. 2002) and the X-ray (Montmerle et al. 1983; Casanova et al. 1995; Grosso et al. 2000; Imanishi et al. 2001). 
From the perspective of our study of molecular outflows, the protostellar stages of star formation are critical. From the near-infrared spectra, Luhman \& Rieke (1999) conclude that roughly $17 \%$ of the YSOs are Class I sources, which implies a lifetime of about $0.1 \mathrm{Myr}$ for this stage and the availability of over 20 potential driving sources for powerful molecular outflows. In order to detect the outflows in $\rho$ Oph, a range of surveys at millimetre, infrared and optical wavelengths have been undertaken. Some $15 \mathrm{CO}$ outflows have so far been recorded, where the most prominent examples arise from IRS 44 (Terebey et al. 1989), VLA 1623 (Andre et al. 1990; Dent et al. 1995) and GSS 30 (Tamura et al. 1990). The earlier studies have recently been complemented by the more systematic search from Bontemps et al. (1996), who detected a total of $11 \mathrm{CO}$ outflows in the region. In addition, Sekimoto et al. (1997) found four CO outflows which arise from X-ray emitting protostars and Kamazaki et al. (2003) have added a further three $\mathrm{CO}$ outflows.

Atomic jets and Herbig-Haro (HH) objects have been discovered through optical searches at the perimeter of the cloud (Wilking et al. 1997; Gómez et al. 1998; Phelps \& Barsony 2004) and at large distances from the cloud core (Wu et al. 2002). The optical studies suggest that the outflows extend over scales up to one degree. Wu et al. (2002) discovered seven groups of $\mathrm{HH}$ objects 2-3 pc away from the rho Oph dark cloud core. Among these, the three brightest objects, HH 549, HH 550, and HH 551, display characteristic HH morphologies of a bow shock or knot with wings or a tail. HH 550 and HH 551 are located more than $1 \mathrm{pc}$ away from the cloud and possibly form a parsec-scale HH flow in Ophiuchus. It is plausible that optical emission lines from embedded flows in the denser clumps are not observed because of the large extinction of $A_{\mathrm{V}}=50-100 \mathrm{mag}$ in the clumps (Wilking \& Lada 1983).

In contrast, near-infrared $\left(\mathrm{H}_{2} 2.12 \mu \mathrm{m}\right)$ imaging observations have detected and explored shock-excited knots and jets located in highly obscured areas (Davis \& Eisloeffel 1995; Dent et al. 1995; Davis et al. 1999; Grosso et al. 2001). These searches have been confined to small regions, typically $5^{\prime} \times 5^{\prime}$. However, Gómez et al. (2003) presented the results from nearinfrared and optical surveys covering a large portion of the $\rho$ Oph cloud. They chose three partly adjoining regions with a combined area of 480 square arcminutes to survey in the near infrared with the New Technology Telescope (NTT). With an integration time of $480 \mathrm{~s}$, many new $\mathrm{H}_{2}$ emission knots which could constitute 13 distinct $\mathrm{H}_{2}$ outflows were detected.

However, the close distance to the $\rho$ Oph molecular cloud, in addition to the optical work of Wu et al. (2002), suggests that typical flow extensions in $\rho$ Oph are of the order of one degree (i.e. a linear flow extent of $2 \mathrm{pc}$ at a distance of $130 \mathrm{pc}$ ). Therefore, we need an unbiased search over a field of 1 square degree in order to carry out a complete census of the molecular outflows in the $\rho$ Oph cloud and to determine their connection to the driving sources. Our initial intention was indeed to survey a field this size in both the near-infrared and millimetre wavelength regions. This goal was achieved in our millimetre survey, which is described in Stanke et al. (2004, Paper II). Unfortunately, weather losses and an insufficient allocation of

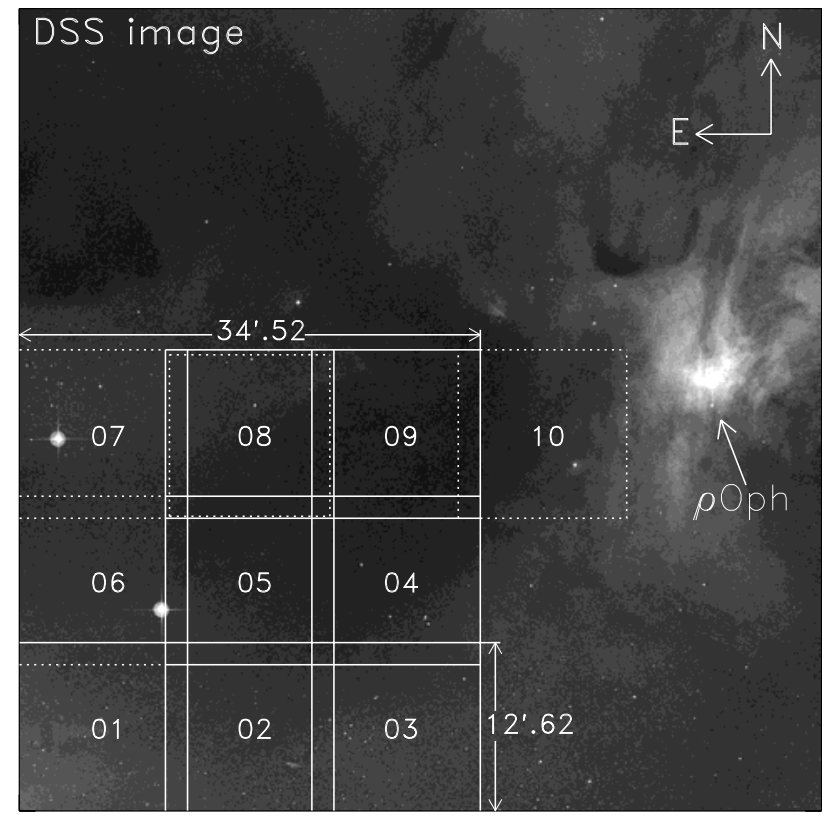

Fig. 1. The scheme of the survey carried out in the $\mathrm{H}_{2}$ filter overlaid on the DSS $1 \times 1$ degree image. Fields 01 to 10 are labelled here to show the central positions (see Table 1). Solid line boxes indicate the part of the region surveyed with the NTT and dotted line boxes show the supplementary survey using the CAHA $3.5 \mathrm{~m}$.

time resulted in a significantly smaller area being surveyed in the near-infrared. The near-infrared survey was conducted in August 2001 using SOFI at the NTT on La Silla. Additional data were secured at high airmass using the OmegaPrime camera at the Calar Alto $3.5 \mathrm{~m}$ telescope. The result is an unbiased $600 \mathrm{~s} \mathrm{H}_{2}$ survey of an area of 1320 square arcmin. The direct purposes of the survey are to determine the sizes and the number of the outflows, and to trace them back to millimetre sources.

\section{Observations and data analysis}

\subsection{Observing strategy}

The area covered by our near-infrared observations is shown superimposed on a DSS ${ }^{1}$ image in Fig. 1. The seven boxes labelled 01, 02, 03, 04, 05, 08, and 09 outline individual fields of 12 ' $6 \times 12$. 6 size which were observed with the near-infrared camera SOFI at the NTT. on La Silla. Data were accumulated during seven first half nights in the period August 2-8, 2001. SOFI is equipped with a $\mathrm{HgCdTe} 1024 \times 1024$ HAWAII detector. We have used a pixel scale of $0.276 \mathrm{arcsec} / \mathrm{pixel}$, which results in an area of $4.7 \times 4$. $^{\prime} 7$ covered in one integration. Images were obtained in the $\mathrm{H}_{2} 1-0 \mathrm{~S}(1)$ narrow band filter, with passband centre at $\lambda=2.124 \mu \mathrm{m}$ and width of $0.028 \mu \mathrm{m}$, and in

\footnotetext{
DSS images were produced at the STSI under US Government grant NAG W-2166. The images of these surveys are based on photographic data obtained using the Oschin Schmidt Telescope on Palomar Mountain and the UK Schmidt Telescope. The plates were processed into the present compressed digital form with the permission of these institutions.
} 
Table 1. List of observed positions shown in Fig. 1 and observing conditions.

\begin{tabular}{|c|c|c|c|c|c|c|}
\hline (1) & (2) & (3) & (4) & (5) & (6) & (7) \\
\hline Observed & RA & Dec & Telescopes $^{\dagger}$ & Integration & Seeing & \\
\hline position & $(2000.0)$ & $(2000.0)$ & used & (s) & (in ") & Noise $^{\star}$ \\
\hline Field01 & $16.28 .44^{\mathrm{s}} . .3$ & $-24^{\circ} 53^{\prime} 42^{\prime \prime}$ & 1 & 600 & 0.8 & 0.76 \\
\hline Field02 & $16.27 .56^{\mathrm{s}} . .1$ & $-24^{\circ} 53^{\prime} 42^{\prime \prime}$ & 1 & 600 & 0.7 & 0.55 \\
\hline Field03 & $16.27^{\mathrm{m}} .07 . .9$ & $-24^{\circ} 53^{\prime} 42^{\prime \prime}$ & 1 & 600 & 1.2 & 0.74 \\
\hline Field04 & $16^{\mathrm{h}} .27^{\mathrm{m}} .07^{\prime} . .9$ & $-24^{\circ} 42^{\prime} 45^{\prime \prime}$ & 1 & 600 & 0.7 & 0.85 \\
\hline Field05 & $16^{\mathrm{h}} .27^{\mathrm{m}} \cdot 56^{\mathrm{s}} . .1$ & $-24^{\circ} 42^{\prime} 45^{\prime \prime}$ & 1 & 600 & 0.6 & 0.94 \\
\hline Field06 & $16.28 \mathrm{~m} .44 . .3$ & $-24^{\circ} 42^{\prime} 45^{\prime \prime}$ & 2 & 540 & 0.9 & 3.79 \\
\hline Field07 & $16^{\mathrm{h}} .28^{\mathrm{m}} \cdot 44^{\mathrm{s}} .3$ & $-24^{\circ} 31^{\prime} 48^{\prime \prime}$ & 2 & 540 & 1.6 & 3.36 \\
\hline Field08 & $16^{\mathrm{h}} \cdot 27^{\mathrm{m}} \cdot 56^{\mathrm{s}} . .1$ & $-24^{\circ} 31^{\prime} 48^{\prime \prime}$ & $1 ; 2$ & $450 ; 270$ & $0.7 ; 0.7$ & $1.55 ; 3.33$ \\
\hline Field09 & $16^{\mathrm{h}} .27^{\mathrm{m}} 07^{\prime} . .9$ & $-24^{\circ} 31^{\prime} 48^{\prime \prime}$ & 1 & 600 & 0.9 & 0.93 \\
\hline Field10 & $16^{\mathrm{h}} .26^{\mathrm{m}} 19^{\mathrm{s}} . .7$ & $-24^{\circ} 31^{\prime} 48^{\prime \prime}$ & 2 & 540 & 1.2 & 3.45 \\
\hline
\end{tabular}

$\dagger$ - Used telescopes: 1 ESO/NTT; 2 CAHA $3.5 \mathrm{~m}$.

$\star-$ noise in units of $10^{-18} \mathrm{~W} \mathrm{~m}^{-2} \operatorname{arcsec}^{-2}$, see Sect 2.1 for explanation.

the $K_{\mathrm{s}}$ broadband filter, with passband center at $2.162 \mu \mathrm{m}$ and width of $0.275 \mu \mathrm{m}$.

Additional data were obtained at the Calar Alto Observatory using the OmegaPrime camera at the $3.5 \mathrm{~m}$ telescope. Note that from Calar Alto, $\rho$ Oph is observable only at airmass greater than 2 at any given time. The resulting noise in the Calar Alto frames is significantly higher than the noise in the SOFI frames (cf. Table 1). The fields covered with OmegaPrime are labelled 06, 07, 08 and 10 and are indicated in Fig. 1 using dotted lines. Field 08 was observed with both SOFI and OmegaPrime. The adopted observing strategy with OmegaPrime was the same as employed with SOFI. The Calar Alto observations were carried out during three first half nights of August 10-13, 2003. Omega Prime (Bizenberger et al. 1998) is equipped with a Rockwell $1024 \times 1024$ pixel HAWAII array detector and provides a fixed pixel scale of $0.4 \mathrm{arcsec} / \mathrm{pixel}$. The total field of view covered in one integration is $6{ }^{\prime} 8 \times 66^{\prime} 8$. Images were obtained in the narrow band $\mathrm{H}_{2} 1-0 \mathrm{~S}(1)$ filter centered at $\lambda=2.125 \mu \mathrm{m}$ and a width of $0.0206 \mu \mathrm{m}$, as well as in the $K_{\mathrm{s}}$ broadband filter, with passband centered at $\lambda=2.196 \mu \mathrm{m}$ and width of $0.388 \mu \mathrm{m}$.

Our observing strategy was to reach a homogeneous integration time over the entire surveyed region. Therefore, the following observing pattern was adopted. For each of our Fields 01-10, an exposure was obtained at the centre of the field, then the subsequent eight exposures were taken with the telescope offset by steps of $229^{\prime \prime}$ in -RA, +Dec, +RA, +RA, - Dec, -Dec, -RA, -RA, where RA and Dec are right ascension and declination, respectively (see Khanzadyan 2003, for more details). The resulting overlap of $70^{\prime \prime}$ is sufficient for accurate mosaicking. Furthermore, at each position, integrations were accumulated adopting a jitter box of 20 arcsec. In this way, a total area of 12!.6 $\times 12$. 6 has been covered per field, and a total connected area of some $34.5 \times 34.5$ has been covered. In general, total integration times per observed position are $600 \mathrm{~s}$ for the $\mathrm{H}_{2}$ filter and $120 \mathrm{~s}$ for the $K_{\mathrm{s}}$ filter. Table 1 lists the right ascension and declination of the fields, the telescope utilised, the total integration time per field, the average seeing as measured on the images, and the noise in the frames, respectively. The noise in the sky-subtracted images was obtained by averaging the mean deviation of the sky in 10 different object-free areas using $10^{\prime \prime}$ apertures.

\subsection{Data reduction}

The data reduction utilised the KAPPA and CCDPACK software developed under STARLINK. Sky frames were constructed from typically 20 object frames and flat fielding was achieved with integrations on a flat field screen, with difference images (lamp on) - (lamp off) used as flats. In the next stage, due to the large amount of data, an automatic registering and mosaicking technique has been employed, using the CCDPACK routines FINDOBJ, FINDOFF, REGISTER, TRANNDF and MAKEMOS. In this manner, the mosaics in all observed Fields have been constructed. Finally the complete observed region mosaics have been constructed in each observed filter by interactive methods provided by the CCDPACK software (PAIRNDF, REGISTER, TRANNDF and MAKEMOSAIC).

\subsection{Astrometric calibration}

The astrometric calibration was performed in several stages. Firstly we calibrated the entire field presented in Fig. 2, where we achieved about 1.5 arcsec accuracy by using DSS and $2 \mathrm{MASS}^{2}$ images to identify the stars, HST guide

\footnotetext{
2 2MASS two micron all sky survey project is collaboration between The University of Massachusetts and the Infrared Processing and Analysis Center (JPL/Caltech).
} 


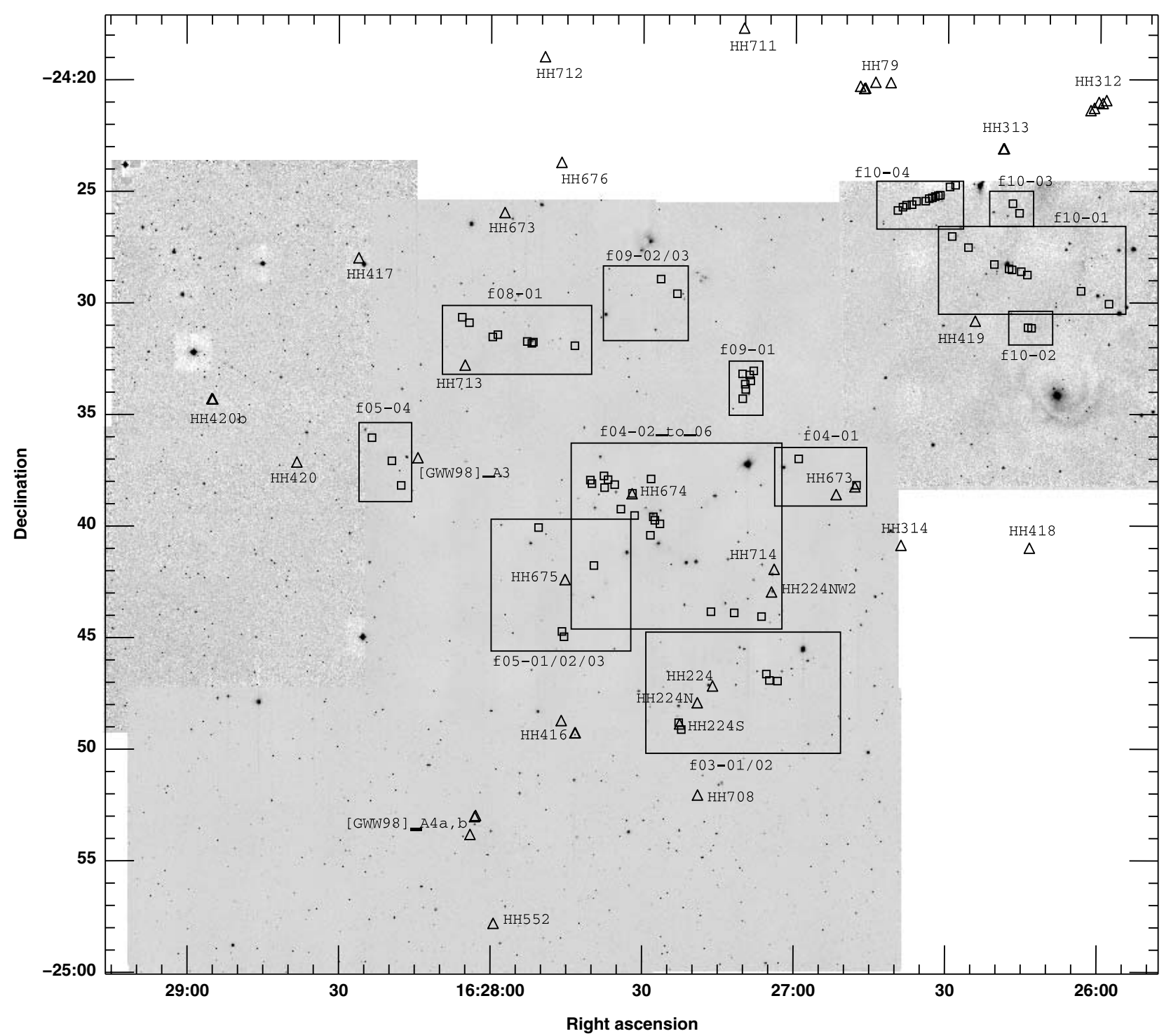

Fig. 2. The portion of the $\rho$ Ophiuchi cloud covered by our survey in $\mathrm{H}_{2} 1-0 \mathrm{~S}(1)$ line + Continuum. New and previously known $\mathrm{H}_{2} 1-0 \mathrm{~S}(1)$ line objects are marked with squares. Optical Herbig-Haro objects are labelled with triangles. The regions demarcated by large boxes are labelled according to the knot definitions explained in the text and are presented further in Figs. 3 to 14.

star catalogue $\left(\mathrm{GSC}^{3}\right)$ and $\mathrm{SIMBAD}^{4}$ data base to determine the coordinate solution. The resulted astrometry is of course affected by field distortions over the SOFI or OmegaPrime fields, but it gave us a basis for further improvements.

In the second stage, parts with already detected $\mathrm{H}_{2}$ emission were separated and astrometrically calibrated again using the same data bases as during the first stage. This time we achieved an accuracy of under 0.5 arcsec mainly due to using small individual fields of 2-3 arcmin, which ensures that any field distortion is small. In this way, the coordinates given in Table 2 (only available in electronic form at the CDS) were obtained.

\footnotetext{
${ }^{3}$ GSC was produced at the STSI under US Government grant. These data are based on photographic data obtained using the Oschin Schmidt Telescope on Palomar Mountain and the UK Schmidt Telescope.

${ }^{4}$ SIMBAD is online database, operated at CDS, Strasbourg, France.
}

\subsection{Continuum subtraction}

The following systematic approach was employed to reliably extract the "pure" $\mathrm{H}_{2} 1-0 \mathrm{~S}(1)$ line emission objects.

- Firstly, the $\mathrm{H}_{2}$ line and $K_{\mathrm{s}}$ images of individual fields were aligned and then blinked with each other ensuring that the stars on both of them have the same brightness on the image display. Objects present on the $\mathrm{H}_{2}$ line image and absent or faint on the $K_{\mathrm{s}}$ band image were counted as a detection. In this manner, most of the objects in our survey were detected. Hereafter, we will refer to this stage as an "eye" detection.

- Secondly, we separated the fields with already detected objects and performed higher accuracy alignment to ensure that the field distortion effect is small or recognizable. Then PSFs (Point Spread Functions) for stars on both images were constructed and compared with each other to determine the scaling factor which was used to 


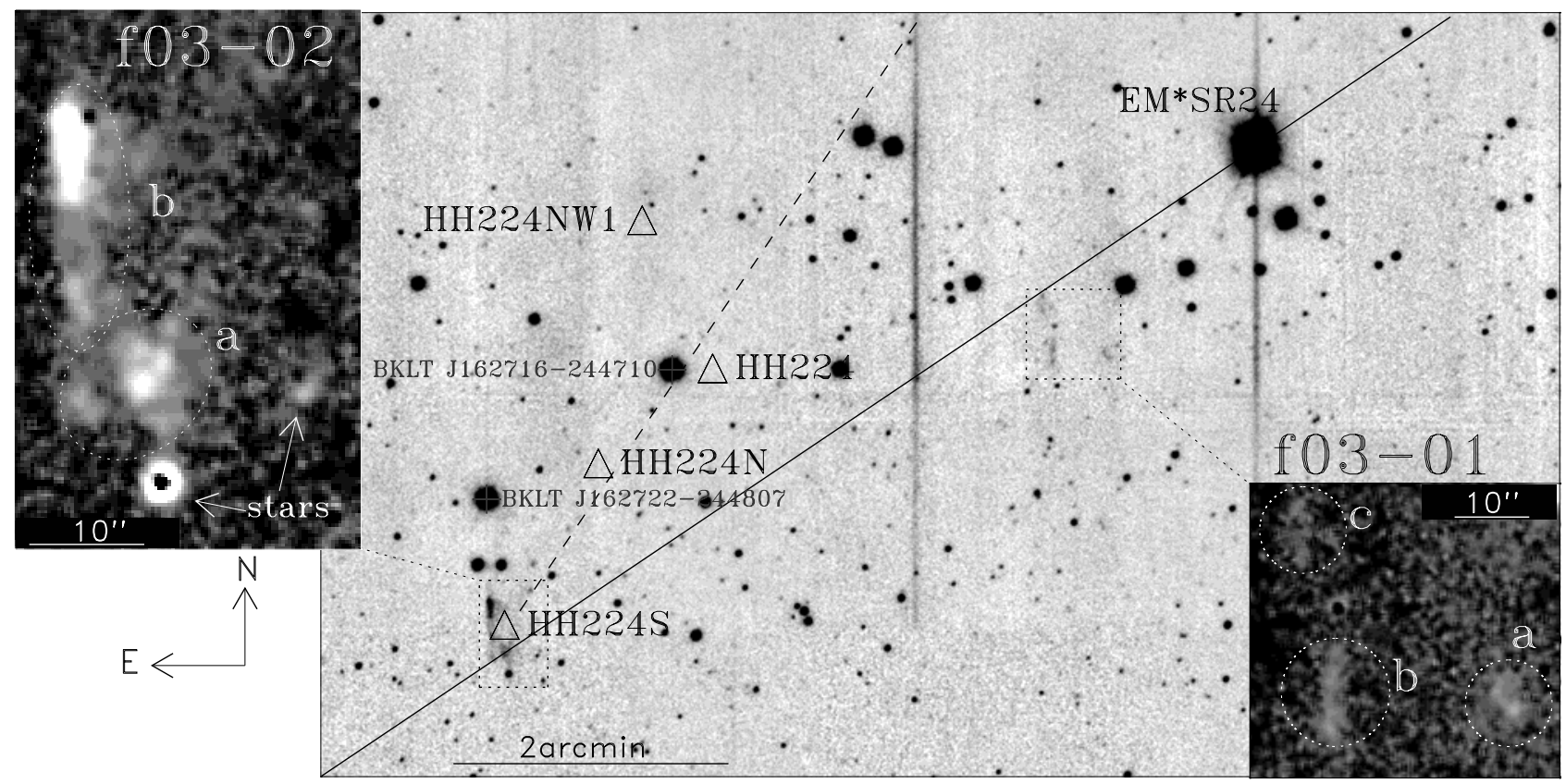

Fig. 3. Knots $f \otimes 3-01$ and $f \otimes 3-02$. The main gray-scale image is $\mathrm{H}_{2} 1-0 \mathrm{~S}(1)$ line + Continuum and the inverted-gray-scale inserts are smoothed pure $\mathrm{H}_{2} 1-0 \mathrm{~S}(1)$ line images, where $\mathrm{H}_{2}$ line emission features are marked with dashed circles and ellipses. The dashed line on the main picture shows the HH 224 flow direction suggested by Phelps \& Barsony (2004) and the solid line shows our suggested flow direction from EM*SR24.

multiply the $\mathrm{H}_{2} 1-0 \mathrm{~S}(1)$ line image. This was followed by the actual subtraction of the $K_{\mathrm{s}}$ band image from the already scaled $\mathrm{H}_{2}$ line image, which enabled us to identify pure $\mathrm{H}_{2}$ 1-0 $\mathrm{S}(1)$ shocked material, but excluded some of the weak "eye" detection.

- Thirdly, before subtracting the $K_{\mathrm{s}}$ band image from the already scaled $\mathrm{H}_{2}$ line image, both images were processed with an IDL based routine LEEFILT originally implemented by Lee (1986), which is an enhancement process based on unsharp masking. Then, after the subtraction, the resulting image was smoothed with a 3-pixel box. In this way, we confirm all "eye" detections and add new ones. Additionally, images in the inserts of Figs. 3-14 are obtained via this method.

This approach gives satisfactory results when identifying pure $\mathrm{H}_{2}$ 1-0 S(1) shocked material but, due to the redder central wavelength of the $K_{\mathrm{s}}$ filter in respect to the $\mathrm{H}_{2}$ line one (see Sect. 2.1), this can cause over-subtraction of the red stars and under-subtraction of the blue stars. Also, due to the fact that the $K_{\mathrm{s}}$ and $\mathrm{H}_{2}$ images are taken in slightly different weather conditions, after subtraction residuals around the stars might still remain.

\subsection{Flux calibration}

The flux calibration of the newly discovered $\mathrm{H}_{2}$ emission objects was obtained via observations of the infrared standard stars Nos. 9155, 9157, 9172, 9175, and 9181 from Persson et al. (1998). After identical data reduction of the science and associated standard frames for each observed field, a specific standard star has been used, which was observed under similar weather conditions. Then using GAIA ${ }^{5}$ we measure integrated counts from the standard in a fixed aperture (usually $2^{\prime \prime}$ is sufficient in our case), then calculate the flux density expected from the standard at $2.12 \mu \mathrm{m}$ and converted it to a flux by multiplying by the filter bandwidth. Finally we calibrate the counts per pixel in each $\mathrm{H}_{2}$ 1-0 S(1) line image via a comparison of the counts per second from the standard star with the stellar flux (Khanzadyan 2003).

\section{Results}

\subsection{Covered area}

The locations of the $\mathrm{H}_{2}$ emission line features are represented by square symbols in Fig. 2. The final $\mathrm{H}_{2}$ image has been constructed from the 10 overlapping survey fields Field01-Field10 using more than 3000 individual frames. In the subsequent sections, we employ the following nomenclature in order to identify objects. We prefix all knots according to their placement in the corresponding survey field. In each field, objects are identified according to increasing right ascension. For example, object $f 10-01$ is situated in Field 10 and located to the West of object $f 10-02$. In cases where individual objects split up into a number of associated emission knots, we use labels a,b,c, etc.

The areas with detected sources are shown in Figs. 3-14. The larger scale images show the narrowband emission without continuum subtraction. For clarity, we use enlarged inserts in each figure which are continuum subtracted images, displaying the pure molecular hydrogen emission in the $1-0 \mathrm{~S}(1)$ line

\footnotetext{
GAIA is a derivative of the Skycat catalogue and image display tool, developed as part of the VLT project at ESO. Skycat is free software under the terms of the GNU copyright.
} 


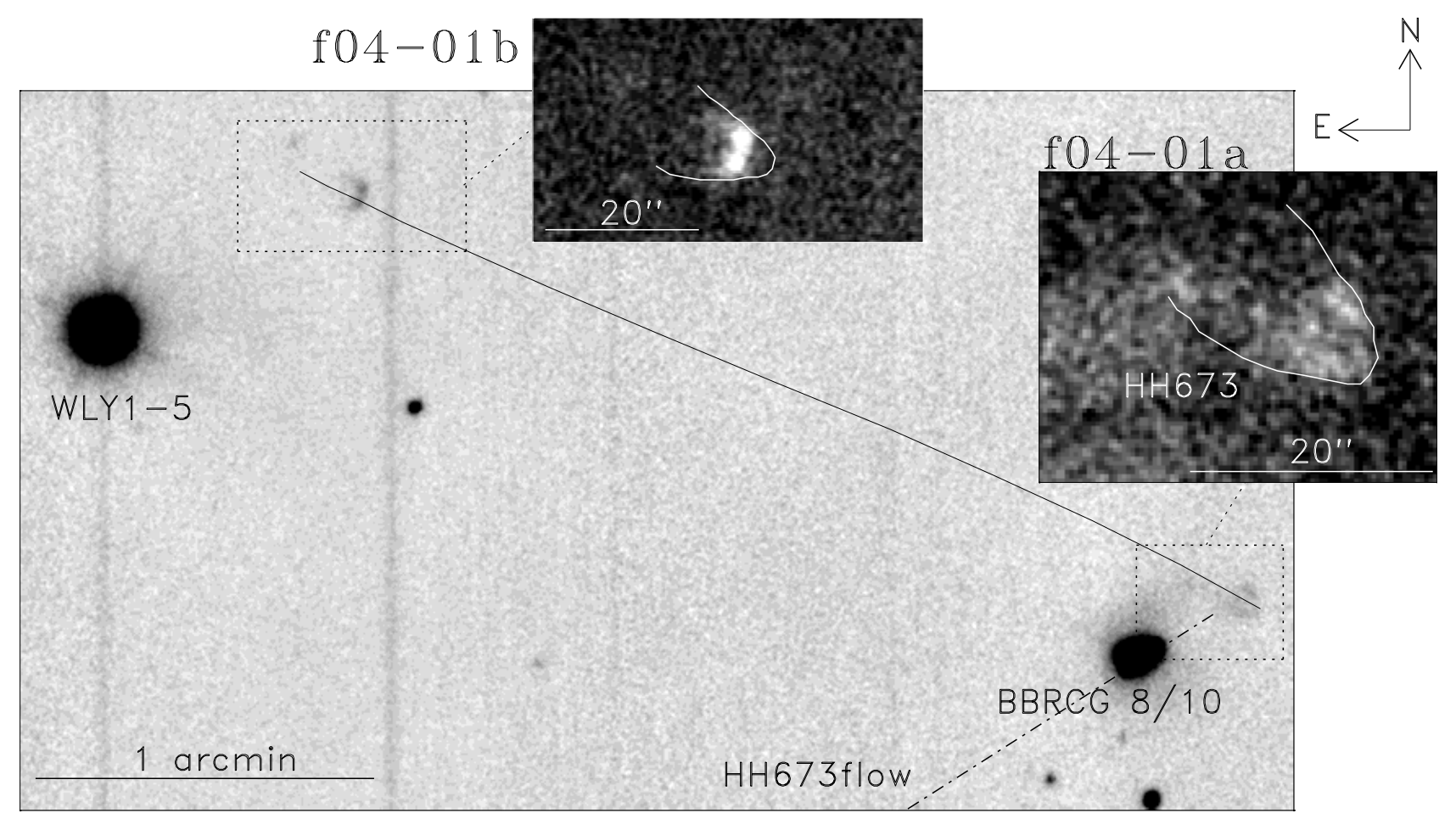

Fig. 4. Flow $f\left(04-01\right.$. The main gray-scale image is $\mathrm{H}_{2} 1-0 \mathrm{~S}(1)$ line + Continuum and the inverted-gray-scale inserts are smoothed pure $\mathrm{H}_{2}$ 1-0 S(1) line images, where $\mathrm{H}_{2}$ line emission features are marked with solid lines in order to emphasize the bow shapes. The solid line on the main image shows the suggested direction of the flow and the dash-dotted line indicates the HH 673 flow direction as was suggested by Phelps \& Barsony (2004). The position of the HH 673b knot is outside of our image along the dash-dotted line.

(see Sect. 2.4). Each figure contains at least one known source which is labelled according to the following nomenclature:

- BKLT: sources from Barsony et al. (1997);

- WL: sources from Wilking \& Lada (1983)

YLW: sources from Young et al. (1986)

WLY: sources from Wilking et al. (1989);

- BBRCG: sources from Barsony et al. (1989).

Table 2 (only available in electronic form at the CDS) summarises the details of the $\mathrm{H}_{2}$ objects found in our survey. The second and third columns list the coordinates of each knot, the fourth and fifth column list the fluxes in units of $10^{-18} \mathrm{~W} \mathrm{~m}^{-2}$ and the corresponding aperture. In several cases, elliptical apertures have been used to derive fluxes. Column 6 cross-identifies our objects with previous detections (Dent et al. 1995; Gómez et al. 2003; Khanzadyan 2003).

\subsection{Field01 and Field02}

We were unable to identify $\mathrm{H}_{2}$ emission knots in Field01 and Field02 down to surface brightnesses of $\sim(0.55-0.76) \times$ $10^{-18} \mathrm{~W} \mathrm{~m}^{-2} \operatorname{arcsec}^{-2}$ in 10 arcsec circular aperture (see Table 1). This is despite the fact that the recent optical surveys (cf. Phelps \& Barsony 2004) report the presence of a number of $\mathrm{HH}$ objects, including HH 416, HH 552, HH 708, and HH 709. The DSS image (see Fig. 1) suggests that the extinction of the cloud in this region is low compared to say Field05 or Field09. The environment may be similar to that of $\mathrm{HH} 34$, where the optical emission also lacks a pronounced near-infrared counterpart (Reipurth et al. 1986). Thus, the optically detected $\mathrm{HH}$ objects in Field01 and Field02 may trace emission which arises in predominantly atomic gas of quite low density.

\subsection{Field03}

Figure 3 shows a section of Field03 where new $\mathrm{H}_{2}$ emission knots have been detected. The two continuum subtracted inserts for $f \otimes 3-\otimes 1 a, b, c$ and $f \otimes 3-\otimes 2 a, b$ display the morphology of the $\mathrm{H}_{2}$ emission. The group $\mathrm{f} 03-02$ corresponds to the optically detected Herbig-Haro object HH 224S (Phelps \& Barsony 2004). However, we do not detect $\mathrm{H}_{2}$ emission from $\mathrm{HH} 224$ and $\mathrm{HH} 224 \mathrm{~N}$ which are indicated in Fig. 3 with triangles. Phelps \& Barsony (2004) stipulate that HH224S is part of a flow which is shown by a dashed line in Fig. 3. We note, however, that HH709, HH 224, f03-01a,b,c, f03-02a,b, and $\mathrm{HH} 418^{6}$ are aligned along a NW-SE direction (indicated by a solid line) which passes through EM*SR 24 (Struve \& Rudkjøbing 1949; Wilking et al. 1989). The star EM*SR 24 (WLY 1-3) coincides with our millimetre source MMS1 of Stanke et al. (2004, Paper II). Alternatively, we note that objects $f \otimes 3-01 a, c, f \otimes 5-01$ and $f \otimes 5-03^{7}$ are aligned in the NE-SW direction. Our millimetre survey does not, however, identify a dense dust core along the latter flow

${ }^{6}$ HH 709 and HH 418 are outside the bounds of Fig. 3. See Fig. 2 for a broader view.

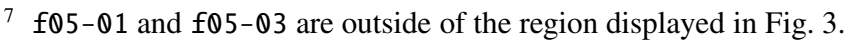
See Figs. 2 and 6 for broader views. 

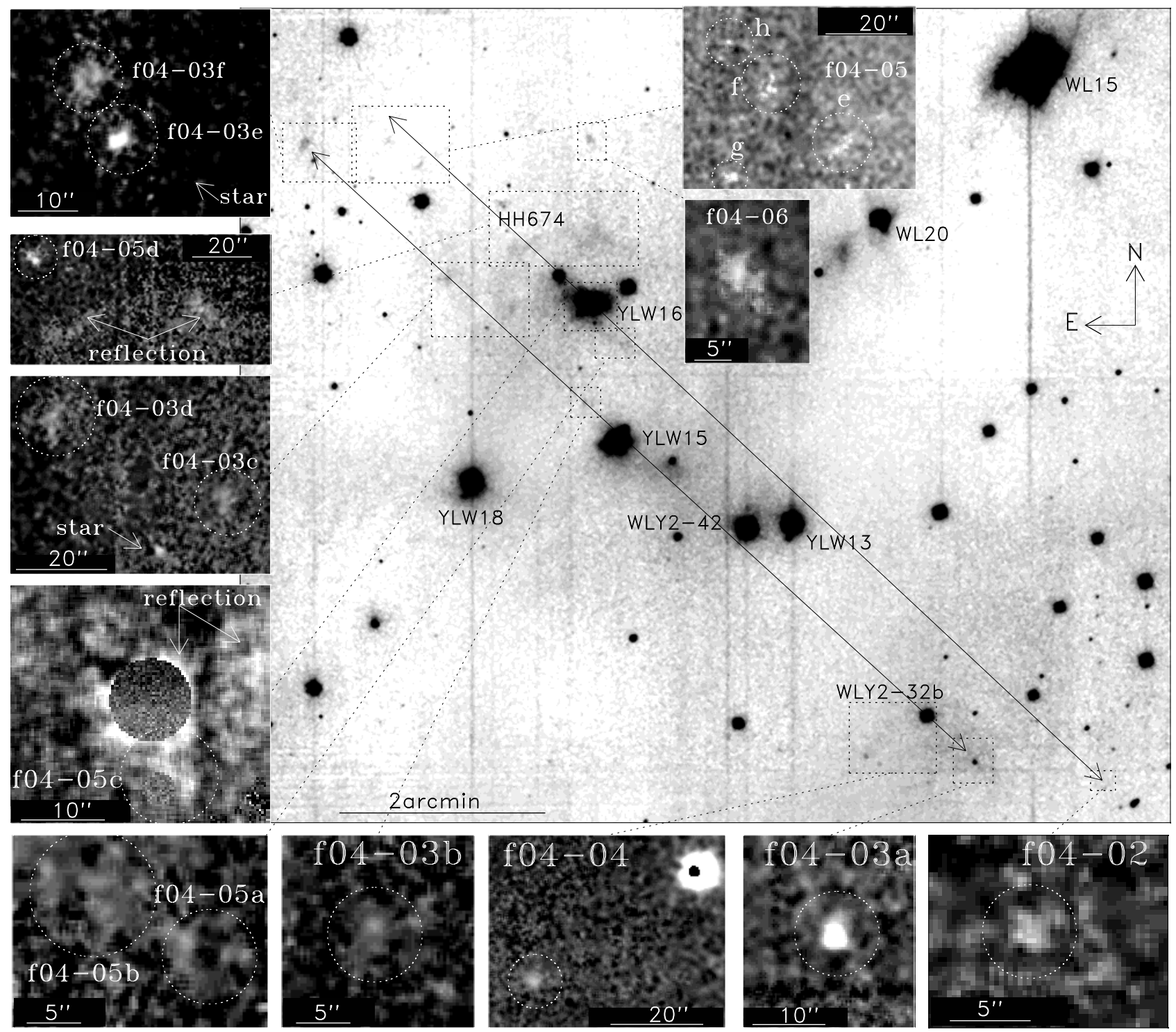

Fig. 5. The region overlapping Field04 and Field05 with knots f04-02 to f04-06. Arrows directed from YLW 15 and YLW 16 indicate the proposed flows. Main gray-scale image is $\mathrm{H}_{2} 1-0 \mathrm{~S}(1)$ line + Continuum and the inverted-gray-scale inserts are smoothed pure $\mathrm{H}_{2} 1-0 \mathrm{~S}(1)$ line images, where $\mathrm{H}_{2}$ line emission features are marked with dashed circles and ellipses.

direction. (As noted earlier, the appearance of stars in the $\mathrm{H}_{2}$ inserts for $f 03-02$ is due to the variable seeing in the $\mathrm{H}_{2}$ and $K_{\mathrm{s}}$ filters.)

\subsection{Field04}

\subsection{1. f04-01}

Figure 4 shows objects $f 04-01 a$ and b of Field04. Both features possess a pronounced bow shock shape with a flow direction pointing towards the south-west. The fQ4-01a structure coincides with the position of HH673 (Phelps \& Barsony 2004). A suggestion here is that HH 673 is linked with $\mathrm{HH} 673 \mathrm{~b}$, (not detected in $\mathrm{H}_{2}$ line and situated outside of our image) forming a flow in the NW-SE direction. That flow direction is indicated by the dash-dotted line in Fig. 4. However, because of the pronounced bow shapes of both $f \otimes 4-02 a$ and $f 04-01 b$, we infer that they arise from a flow connecting both knots with a flow direction represented by the solid line. Both knots appear in the prolongation of the f08-01 flow which is located to the NE (cf. Sect. 3.7 and Fig. 8). Two Herbig-Haro objects, HH 417 in the NE and $\mathrm{HH} 418$ in the south-west, appear along the projection of our suggested flow direction as evident in Fig. 2. They were not detected in the $\mathrm{H}_{2}$ line. The flow, if confirmed, would stretch half a degree.

\subsection{2. f04-02 to and f04-06}

Figure 5 shows a region which overlaps with Field04 and Field05. It contains a total of 17 new $\mathrm{H}_{2}$ emission line objects. Part of this region has already been observed by Gómez et al. (1998), who detected the Herbig-Haro object (GWW98) A2 (later renamed as HH 674 by Phelps \& Barsony 2004). Our observations yield two major flows from YLW 16 


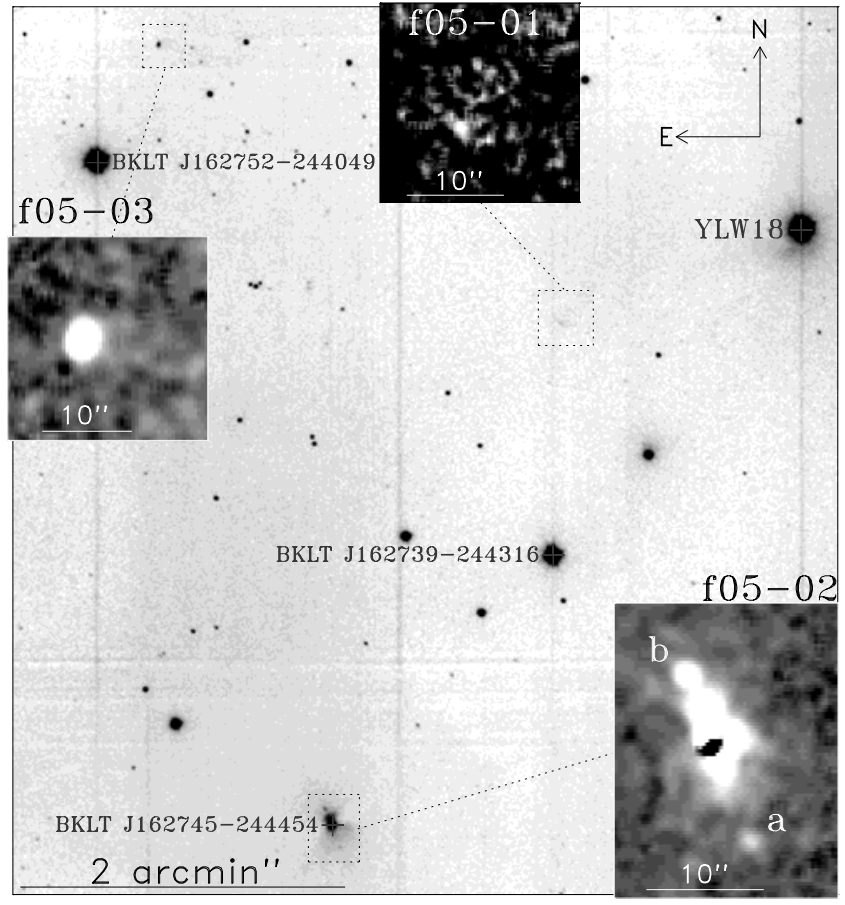

Fig. 6. Part of Field05 where knots $f(05-01, f 05-02$ and $f 05-03$ are situated. Main gray-scale image is $\mathrm{H}_{2} 1-0 \mathrm{~S}(1)$ line + Continuum and the inverted-gray-scale inserts are smoothed pure $\mathrm{H}_{2} 1-0 \mathrm{~S}(1)$ line images.

and from YLW 15. The two proposed flows seem to be parallel on the sky. f04-02, f04-05a, b, f04-05d, and f04-5e, f, h lie along one axis that runs through YLW16, while $f 04-03 a$, f04-03b, f04-03c, d, and f04-03e, f lie along a second flow axis centred on YLW15. These two flow axes are marked with solid line arrows in Fig. 5.

To the south-west of the YLW 15, the point-like object $f \otimes 4-02$ is detected at $2 \sigma$ above the noise in the sky subtracted image of Fig. 5. Following the stipulated flow direction from YLW 15, we see the point-like objects $f 04-03$ a to the SW of YLW 15, and $f 04-03 b, f 04-03 c$ and $d, f 04-03 e$ and $f$ towards the NE. Towards the immediate SW of YLW 16, we identify objects $f(04-05 a, b, c$ and towards the NE, objects $f 04-05 d$ and $f 04-05 e-h$. The position of object $f 04-05 d$ agrees with $\mathrm{HH} 674$.

Located somewhat off the NE-SW flow direction from YLW 15 is f04-04, and north of YLW 16 f04-06 appears. The feature $f \otimes 4-04$ is located some 40 arcsec to the SE of WLY 2-32b and the possibility exists that the two are related. Alternatively, we note that $f(04-04$ and $f 04-06$ are roughly diametrically opposed to WLY 2-42.

The continuum subtracted images show remnant emission around the young stellar object YLW 16 which arises from a reflection nebulae. Imaging polarimetry measurements of the YSO obtained by Lucas \& Roche (1998) (their Fig. 5) reveals the dust distribution in the bipolar cavity of the YLW 16 core region. Our millimetre survey (cf. Paper II: Stanke et al. 2004) results in the detection of several millimetre sources along the NE-SW flows from YLW 15 and YLW 16.

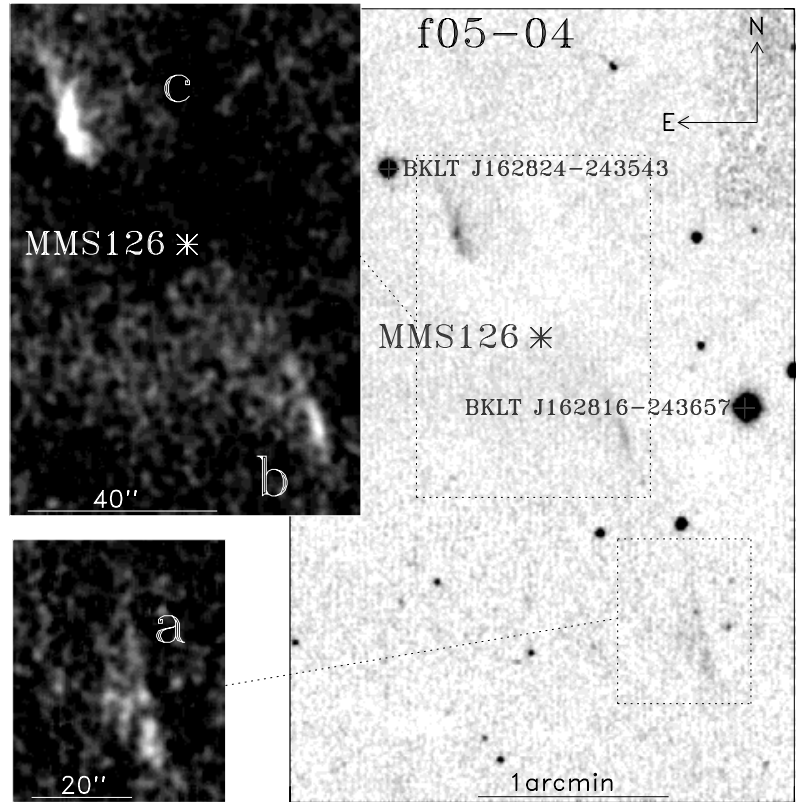

Fig. 7. The f05-04 flow. Main gray-scale image is $\mathrm{H}_{2} 1-0 \mathrm{~S}(1)$ line + Continuum and the inverted-gray-scale inserts are smoothed pure $\mathrm{H}_{2}$ 1-0 S(1) line images. The position of the MMS126 source from our Stanke et al. (Paper II: 2004) is marked with an asterisk.

\subsection{Field05}

\subsection{1. f05-01 to f05-03}

Figure 6 shows the newly discovered $\mathrm{H}_{2}$ emission objects $f \otimes 5-01, f \otimes 5-02$, and $f 05-03$, located in Field05. Object fQ5-01 has a morphology which is reminiscent of a bow shock pointing towards the SE or SW. Because of its proximity to YLW 15 and YLW 16 (about $3^{\prime}$ to the NW), it may very well arise from a NW-SE flow from this region, and possibly be connected to $f \otimes 9-01$ (see Fig. 2). Object $f \otimes 5-03$ has an elongated shape with the large semi-major axis pointing towards YLW 16. However, it is intriguing to speculate that $f 05-01, f 05-03$, and $f 03-01 a, c$ form part of a gigantic flow which extends from the NE to SW (cf. Fig. 2 and Sect. 3.3). This possibility will be further discussed by Smith et al. (2004, Paper III).

Knots $f 05-02 a$ and b, shown in the second insert of Fig. 6, are diametrically opposed to BKLT J162745-244454 and located in the immediate vicinity of this object. We purport they arise from an edge-on disk system with a bipolar outflow. The authenticity of the knots was proved by blinking the continuum image with the line image as it is explained in Sect. 2.4. The residuals around the star BKLT J162745-244454 are present simply due to the fact that the shape of the PSF changes due to field rotation, e.g., diffraction patterns from the secondary support rotate when observing with an alt-azimuth telescope.

\subsection{2. $f 05-04$}

Figure 7 shows the three extended $\mathrm{H}_{2}$ emission knots f05-04a-c which is one of the most intriguing flows discovered in our survey. It consists of several extended emission 

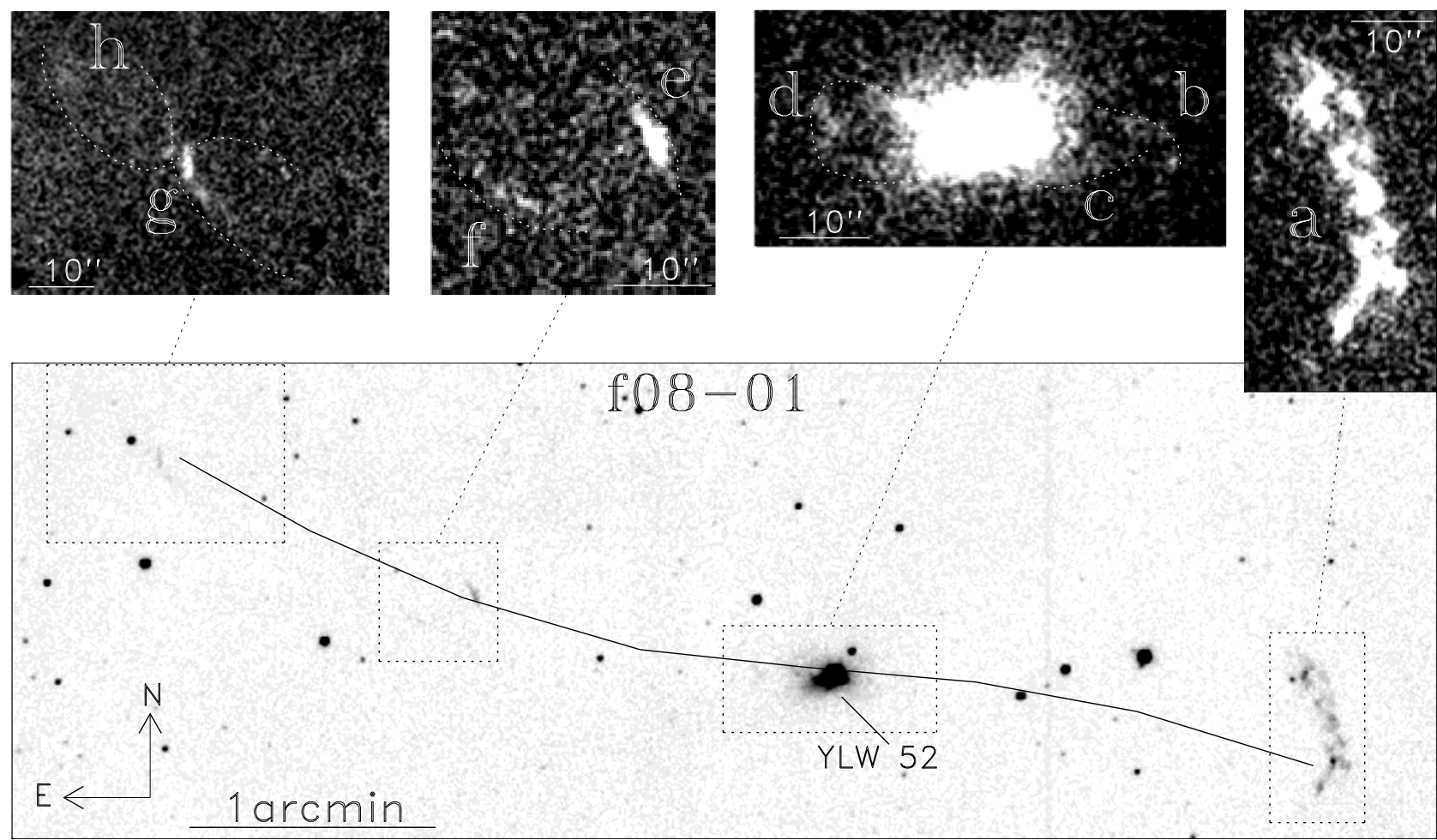

Fig. 8. Flow $f 08-01$. The solid line indicates the suggested $\mathrm{S}$-shaped outflow. Main gray-scale image is $\mathrm{H}_{2} 1-0 \mathrm{~S}(1)$ line $+\mathrm{Continuum}$ and the inverted-gray-scale inserts are smoothed pure $\mathrm{H}_{2} 1-0 \mathrm{~S}(1)$ line images.

features which are aligned in the NE to SW direction. Most interestingly, our millimetre survey (Paper II: Stanke et al. 2004) has resulted in the discovery of a Class 0 source, MMS126, located right between knots $b$ and c. The position of MMS126 is marked by the asterisk in Fig. 7. We do not find additional emission objects in the north-eastern or south-western prolongation of the flow, which suggests that either the flow is very young indeed or that the environment is diffuse.

\subsection{Field06 and Field07}

We do not detect $\mathrm{H}_{2}$ emission objects in Field06 and Field07 down to the sensitivity limits of $3.3-3.8 \times 10^{-18} \mathrm{~W} \mathrm{~m}^{-2} \operatorname{arcsec}^{-2}$ in a 10 arcsec circular aperture (see Table 1). Both fields contain a number of optical $\mathrm{HH}$ objects including $\mathrm{HH} 420$, $\mathrm{HH} 420 \mathrm{~b}$, and HH 417. Because observations of Field06 and Field07 were carried out from Calar Alto at very high airmass, the noise is significantly higher than in the other fields (cf. Table 1). In addition, the extinction particularly in Field06 could be rather high, as judged from e.g. Fig. 1. The nondetection of near-infrared emission may then result from the excessive noise. Alternatively, the optical $\mathrm{HH}$ objects arise at the near-side of the cloud and trace emission which arises in less dense material, in a medium which may not be dense enough to shock-excite $\mathrm{H}_{2}$ emission.

\subsection{Field08}

We discover $7 \mathrm{H}_{2}$ 1-0 S(1) line excited knots in Field08, as shown in Fig. 8. Knots f08-01a and b are situated west from the source YLW 52 and knots $f 08-01 d$ to $h$ are in the east. YLW 52 is a Class I protostar and exhibits a featureless $K$-band spectrum (Greene \& Lada 2000). Features f08-01e, $f$ and $g$, $\mathrm{h}$ appear to arise from the walls of a cavity, possibly excited by oblique shocks in an S-shaped flow elongated almost E-W. The stipulated flow direction is represented by the solid line in Fig. 8. We note that the objects $f 09-01$ are located at the southwestern prolongation of the flow direction (see Sect. 3.8.1).

Object $f 08-01$ a has been studied by Grosso et al. (2001), who discussed the possibility that $f 08-01$ a arises from two interacting flows which originate from YLW 52 and from YLW 15, (situated outside the bounds of Fig. 8, roughly southwest from $\mathrm{f} \otimes 8-01 \mathrm{a}$ and is separated by about $8 \mathrm{arcmin}$ ).

\subsection{Field09}

\subsection{1. f09-01}

Figure 9 shows knots $f 09-01 \mathrm{a}-\mathrm{g}$ of Field09. The knots f09-01a-f were discovered by Gómez et al. (2003), displayed in their field (GSWC2003)3. BBRCG 27 (WL 10) is a Class II source from which a $\mathrm{CO}$ outflow emerges (Sekimoto et al. 1997). Gómez et al. (2003) suggested that the emission arises from multiple flows which overlap. Their conclusion is based on the fact that the region contains a Class I (WL15) and several Class II sources (WL11, WL14, WL21).

Our study results in the detection of an additional knot f09-01g, which coincides with the red lobe of the ${ }^{12} \mathrm{CO}$ outflow detected by Sekimoto et al. (1997). We note that knots $f 09-01 b$ and $d$ are on the same axis as the flow arising 


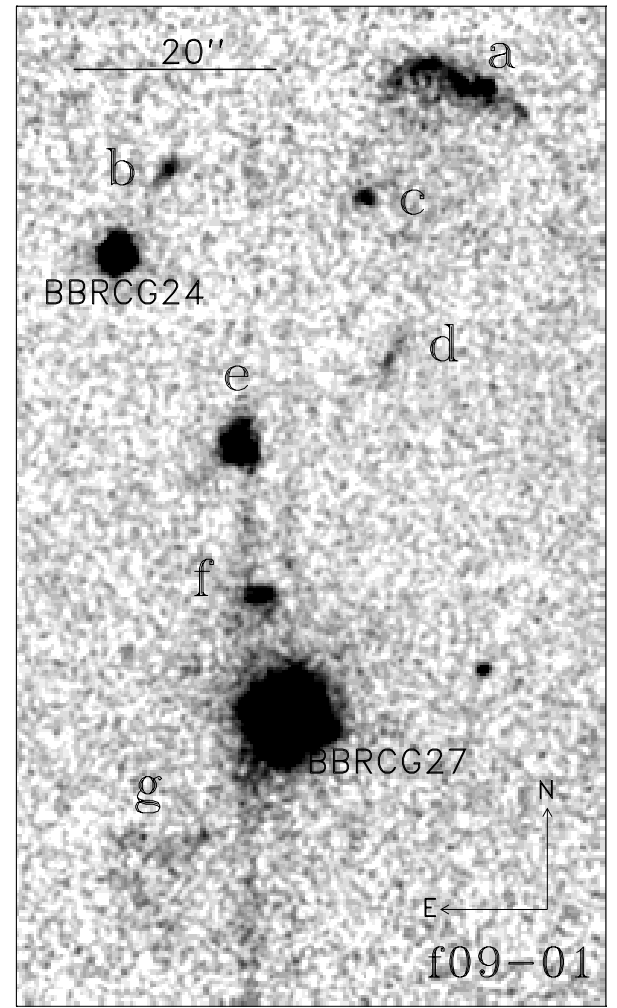

Fig. 9. Flow $f \otimes 9-\otimes 1$ in $\mathrm{H}_{2} 1-0 \mathrm{~S}(1)$ line + Continuum.

from WL 6. This flow forms $f 09-02$ (cf. Sect. 3.8.2), which is located SW of the $f 09-01$ knots. We also note that the bow-shape morphology of $f 09-01 a$ and the location of knots f09-01a, c, and e suggest a NW-SE separate and unrelated flow direction which, if followed towards the SE, may very well generate the bow of $f 05-01$. In this respect, the suggestion of Gómez et al. (2003) that the emission knots in (GSWC2003)3 or $\mathrm{f} \otimes 9-01$ arise from physically distinct flows is supported.

Alternatively, it is worth mentioning that the $f 09-01$ knots might well originate from one flow if we consider a fast precession jet mechanism as discussed by Rosen \& Smith (2004), where some simulations of the $\mathrm{H}_{2} 1-0 \mathrm{~S}(1)$ line emission show a great similarity to the picture we have here.

\subsection{2. f09-02 and f09-03}

Figure 10 shows part of Field09 where knots f09-02 and $\mathrm{f} 09-03$ are located. Both knots were discovered by Gómez et al. (2003). The two inserts in Fig. 10 show nicely the bow like morphologies of both knots. Object f09-02 has a bowlike structure which suggests a flow direction to the NE. Its morphology strongly suggests that it originates from a flow from WL 6 (Wilking \& Lada 1983). The stipulated flow direction is represented by straight lines and arrows in Fig. 10. This conclusion is in agreement with Gómez et al. (2003). On the other hand, the pronounced bow-shock morphology of f09-03 suggests a flow direction towards the NW and that WLY 2-48 is the driving source. The separation of $3^{\prime}$ corresponds to an extent of $0.11 \mathrm{pc}$ at an adopted distance of $130 \mathrm{pc}$. All this suggests that $f 09-02$ and $f 09-03$ are independent flows.

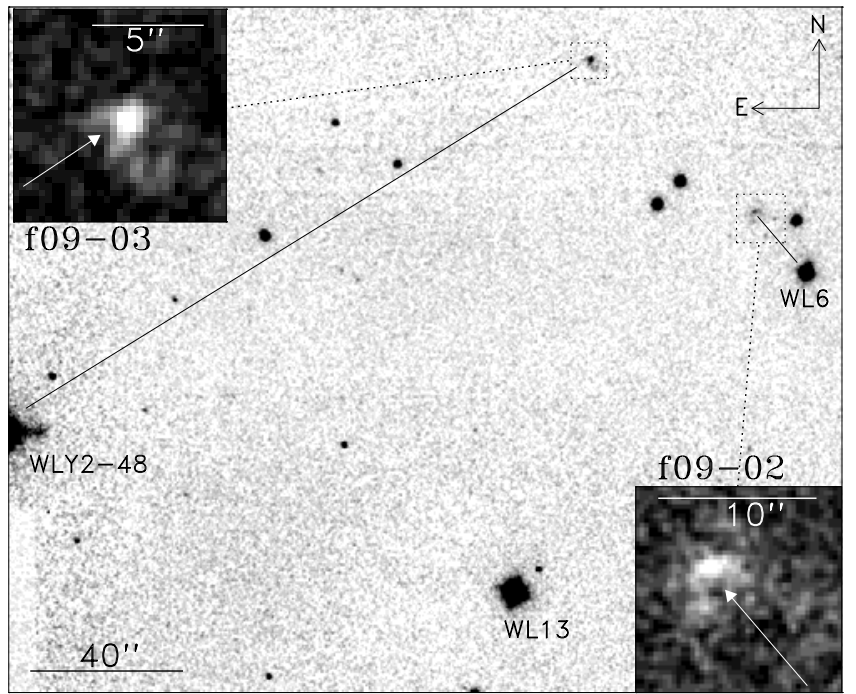

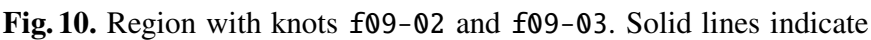
the proposed source and knot connections. Main gray-scale image is $\mathrm{H}_{2}$ 1-0 S(1) line + Continuum and the inverted-gray-scale inserts are smoothed pure $\mathrm{H}_{2}$ 1-0 S(1) line images.

\subsection{Field10}

\subsection{1. $f 10-01$}

Figure 11 shows the chain of nine $\mathrm{H}_{2}$ emission line knots f10-01a-g which extends in the NE-SW direction from YLW 31. The stipulated flow direction is given by a straight line in Fig. 11. The flow extends over a distance of $7.7 \mathrm{arcmin}$ or $0.3 \mathrm{pc}$. We propose YLW 31 as the driving source because of the accurate alignment of the individual knots with respect to this Class II source (Bontemps et al. 2001).

\subsection{2. $f 10-02$}

Figure 12 shows the isolated object $f 10-02$ whose bow-type morphology suggests a flow direction towards the NE. The flow direction is indicated in the insert of Fig. 12. The emission pattern suggests that knot a is a part of a fading wing of the bow and knot $b$ arises from the apex of the bow. The purported flow direction towards the NE is supported by the location of HH 419 (outside of the picture) some 3 arcmin towards the eastern prolongation (see Fig. 2).

\subsection{3. $f 10-03$}

Figure 13 shows a group of knots situated in the upper outskirts of Field10 which we name f10-03 (see Fig. 2). The shape of the bow-like feature, clearly seen on the insert frame as knot b, suggests that the flow originates from a general northern direction. A NE-SW direction is suggested by the location of HH 79 and $\mathrm{HH} 711$ about 9 and 14 arcmin respectively towards the north-east as can be seen in Fig. 2. 


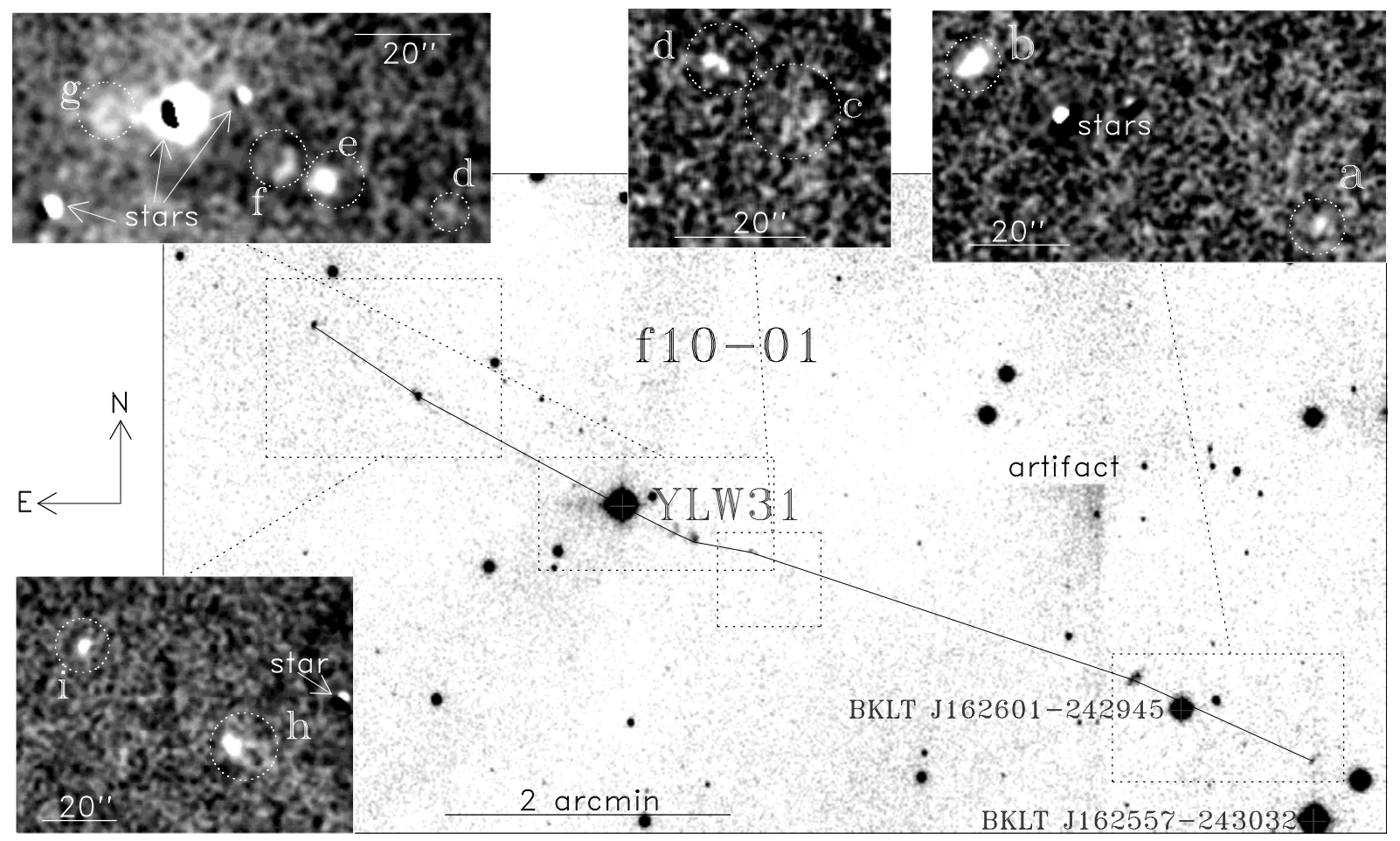

Fig. 11. Flow $f 10-01$. Solid line shows the extent of the proposed flow. Main gray-scale image is $\mathrm{H}_{2} 1-0 \mathrm{~S}(1)$ line + Continuum and the inverted-gray-scale inserts are smoothed pure $\mathrm{H}_{2} 1-0 \mathrm{~S}(1)$ line images.

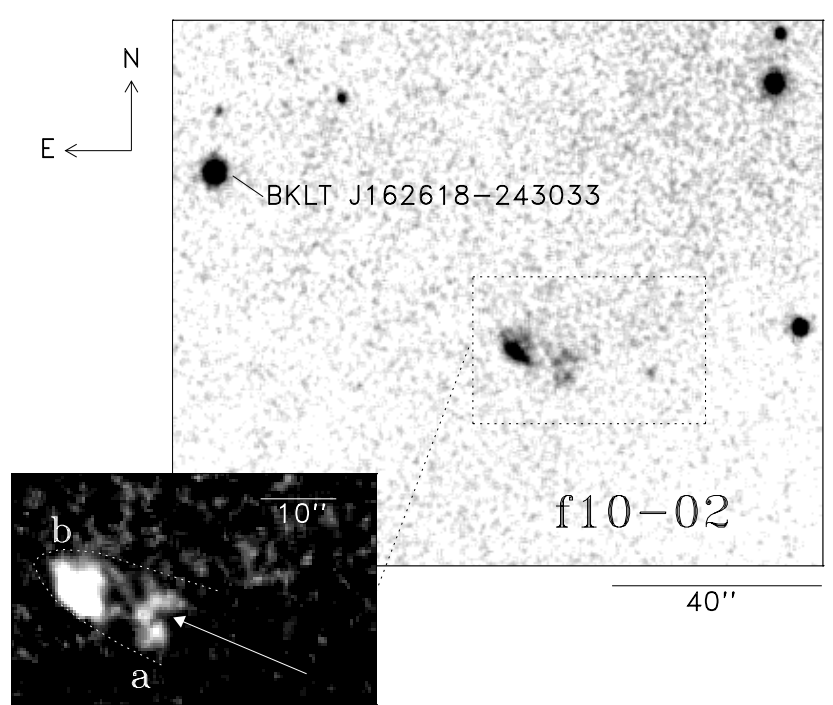

Fig. 12. The bow structure of $f 10-02$. Main gray-scale image is $\mathrm{H}_{2}$ 1-0 S(1) line + Continuum and the inverted-gray-scale insert is smoothed pure $\mathrm{H}_{2} 1-0 \mathrm{~S}(1)$ line image.

\subsection{4. $\mathrm{f} 10-04$}

Figure 14 shows a group of knots located in the south-eastern vicinity of VLA 1623 . We assign names $f 10-04 a-m$ to the individual emission knots. The $\mathrm{H}_{2}$ and $\mathrm{CO}$ emission of this region has been studied in detail by Dent et al. (1995), whose knots $\mathrm{H} 1, \mathrm{H} 2$, and $\mathrm{H} 3$ correspond to $\mathrm{f} 10-04 \mathrm{~h}, \mathrm{f} 10-04 \mathrm{~g}$,

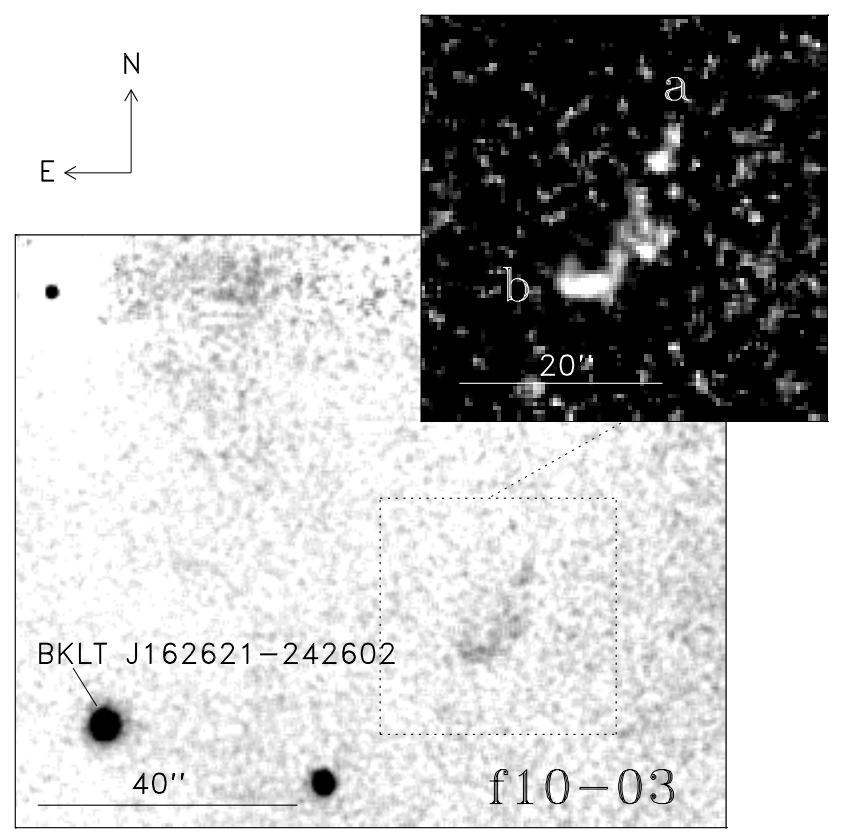

Fig. 13. The $f 10-03$ group of knots. Main gray-scale image is $\mathrm{H}_{2} 1-0 \mathrm{~S}(1)$ line + Continuum and the inverted-gray-scale insert is smoothed pure $\mathrm{H}_{2} 1-0 \mathrm{~S}(1)$ line image.

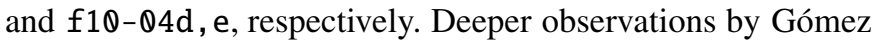
et al. (2003) resulted in the discovery of additional emission which corresponds to our knots $f 10-04 c, f, i, j, 1$. The molecular hydrogen is spatially correlated with the blueshifted CO emission (Andre et al. 1990; Dent et al. 1995) and the solid line drawn in Fig. 14 corresponds to the flow direction. Our observations has uncovered further knots $\mathrm{f} 10-04 \mathrm{a}, \mathrm{b}$ along the 


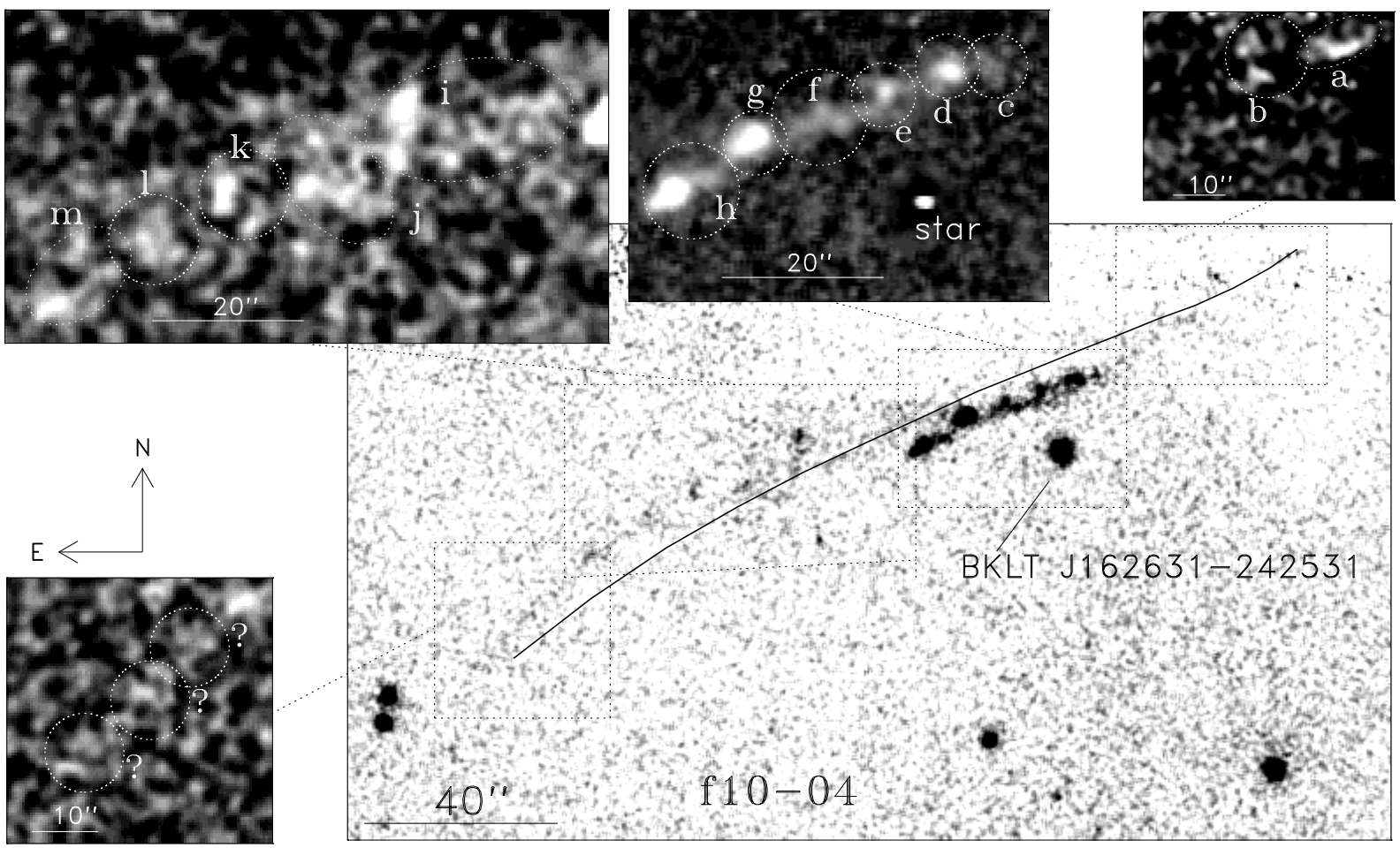

Fig. 14. Flow $f 10-04$. The solid curved line shows the extent of the suggested flow. Main gray-scale image is $\mathrm{H}_{2} 1-0 \mathrm{~S}(1)$ line + Continuum and the inverted-gray-scale insert is smoothed pure $\mathrm{H}_{2} 1-0 \mathrm{~S}(1)$ line image, where $\mathrm{H}_{2}$ line emission features are marked with dashed circles and ellipses.

north-west prolongation of the flow and features at some $1.5 \sigma$ above the background noise in the south-east prolongation, labelled with question marks in Fig. 14. Knots $f 10-04 i-m$ have been identified by Gómez et al. (2003) but were thought to arise from the separate flow (GSWC2003) 21. Our data suggest that all of the knots $f 10-04 a-m$ arise from the VLA 1623 outflow. We note that our fluxes are about a factor of 2 lower than the fluxes of Dent et al. (1995) for knots $f 10-04 \mathrm{~h}, \mathrm{f} 10-04 \mathrm{~g}$, and $\mathrm{f10}-\mathrm{Q} 4 \mathrm{~d}$, e. We suspect inaccurate flux determination from our observations as the observations were obtained at an airmass of 2.2. However, shock waves propagating in jets are predicted to rapidly decay in strength (Smith et al. 1997). Given the small size of the knots and the duration of over nine years between observations, flux variations should be expected. A proper motion of 2 arcsec would ensue given bow shock speeds of $130 \mathrm{~km} \mathrm{~s}^{-1}$ in the plane of the sky. Therefore, monitoring of these features would be illuminating for jet models.

\section{Summary}

We have searched a wide area of the main star-forming cloud in $\rho$ Ophiuchus for emission from vibrationally-excited molecular hydrogen. The emission we detect originates from shock waves since (a) there are no nearby sources of ultraviolet radiation and (b) the emission originates in discrete knots and wings rather than the illuminated rims of dense clumps or pillars. A quantitative analysis, in combination with a detailed comparison to our millimetre survey, will be undertaken in Smith et al. (2004, Paper III, in preparation). Here, we have investigated the links between emission knots and chains of knots in order to constrain the location of driving sources.

The number of distinct protostellar outflows can be estimated from Fig. 2. We find that 10 outflows are sufficient to account for all the $\mathrm{H}_{2}$ emission. Even so, there are alignments which could reduce the number. In fact, if the average size of a protostellar outflow were $0.6 \mathrm{pc}$, (e.g., Stanke 2003), then it would extend 15 arcmin. In other words, the driving protostar may be located far from the molecular hydrogen features. On the other hand, quite compact $\mathrm{H}_{2}$ outflows are also identified (e.g. $f 05-4)$.

The overall distribution of $\mathrm{H}_{2}$ objects follows the network of cloud filaments. This suggests that the outflow components only become detectable when they encounter dense cloud material rather than dragging out and shocking molecular material. Hence, $\mathrm{H}_{2}$ is not a good tracer of outflows that extend beyond the bounds of molecular clouds.

The minimum jet power required to drive the $\mathrm{H}_{2}$ shocks can be estimated here. The integrated 1-0 S(1) $\mathrm{H}_{2}$ fluxes for the flows $f 05-4, f \otimes 8-01$ and $f 10-04$ are $3.4,12.9$ and $29.5 \times$ $10^{-17} \mathrm{~W} \mathrm{~m}^{-2}$. These convert to the very small line luminosities of $1.8,6.8$ and $15.6 \times 10^{-5} L_{\odot}$, respectively, for the distance of $130 \mathrm{pc}$. The total $\mathrm{H}_{2}$ luminosity from the radiating shocked layers is 10-20 times these values (Smith 1995). However, dense hydrodynamic jets are found to be extremely inefficient at thermalising the available power. Hydrodynamic simulations of diverse evolving outflows yield a ratio of jet to 1-0 S(1) luminosity of 300-3000 (Rosen \& Smith 2004). The larger values correspond to highly collimated ballistic flows in which the jet and leading bow shock both move at high speed. The lower 
values correspond to precessing flows. On the other hand, we suspect that a magnetic field cushions the shock, channelling considerably more energy into infrared line emission while, contrarily, the dust extinction tends to screen it out. The result, found to be consistent with the data for protostellar outflows in general (Froebrich et al. 2003), is that jet powers are probably 1000 times the observed 1-0 S(1) luminosity. We thus conclude that driving jet powers are in the range $0.02-0.2 L_{\odot}$.

The majority of outflows are directed into the NE-SW quadrants, transverse to the degree-scale filamentary cloud structure. This could be an intrinsic property or could indicate that extinction limits our ability to detect the outflows. In this context, Kamazaki et al. (2003) found three CO outflows in $\rho$ Oph A all aligned into the NW-SE quadrants with the blue-shifted material directed towards the south-east. Only one of these three $\mathrm{CO}$ outflows is evident in our $\mathrm{H}_{2}$ survey. Hence, although the number of $\mathrm{H}_{2}$ outflows is roughly consistent with both the number of Class 0 and Class I protostars and the number of CO outflows in the same area (Bontemps et al. 1996), a deeper examination is absolutely necessary (Paper III: Smith et al. 2004, in preparation).

Acknowledgements. We acknowledge the data analysis facilities provided by the Starlink Project which is run by CCLRC/Rutherford Appleton Laboratory on behalf of PPARC. The Starlink packages CCDPACK, KAPPA, and GAIA have been used. This work has made extensive use of the SIMBAD database at CDS. T.K. and R.G. benefitted through a Visitor Theory Grant funded by UK's PPARC. MDS is grateful to the MPIA, Heidelberg for hospitality.

\section{References}

Allen, L. E., Myers, P. C., Di Francesco, J., et al. 2002, ApJ, 566, 993 Andre, P., Martin-Pintado, J., Despois, D., \& Montmerle, T. 1990, A\&A, 236, 180

Andre, P., Montmerle, T., \& Feigelson, E. D. 1987, AJ, 93, 1182

Barsony, M., Carlstrom, J. E., Burton, M. G., Russell, A. P. G., \& Garden, R. 1989, ApJ, 346, L93

Barsony, M., Kenyon, S. J., Lada, E. A., \& Teuben, P. J. 1997, ApJS, 112, 109

Benson, P. J., \& Myers, P. C. 1989, ApJS, 71, 89

Bizenberger, P., McCaughrean, M. J., Birk, C., Thompson, D., \& Storz, C. 1998, in Infrared Astronomical Instrumentation, ed. Albert M. Fowler, Proc. SPIE, 3354, 825

Bontemps, S., André, P., Kaas, A. A., et al. 2001, A\&A, 372, 173

Bontemps, S., Andre, P., Terebey, S., \& Cabrit, S. 1996, A\&A, 311, 858

Casanova, S., Montmerle, T., Feigelson, E. D., \& Andre, P. 1995, ApJ, 439, 752

Davis, C. J., \& Eisloeffel, J. 1995, A\&A, 300, 851

Davis, C. J., Smith, M. D., Eislöffel, J., \& Davies, J. K. 1999, MNRAS, 308, 539

de Geus, E. J., Bronfman, L., \& Thaddeus, P. 1990, A\&A, 231, 137

Dent, W. R. F., Matthews, H. E., \& Walther, D. M. 1995, MNRAS, 277, 193
Froebrich, D., Smith, M. D., Hodapp, K.-W., \& Eislöffel, J. 2003, MNRAS, 346, 163

Gómez, M., Stark, D. P., Whitney, B. A., \& Churchwell, E. 2003, AJ, 126,863

Gómez, M., Whitney, B. A., \& Wood, K. 1998, AJ, 115, 2018

Greene, T. P., \& Lada, C. J. 2000, AJ, 120, 430

Grosso, N., Alves, J., Neuhäuser, R., \& Montmerle, T. 2001, A\&A, 380, L1

Grosso, N., Montmerle, T., Bontemps, S., André, P., \& Feigelson, E. D. $2000, A \& A, 359,113$

Imanishi, K., Koyama, K., \& Tsuboi, Y. 2001, ApJ, 557, 747

Johnstone, D., Wilson, C. D., Moriarty-Schieven, G., et al. 2000, ApJ, 545,327

Kamazaki, T., Saito, M., Hirano, N., Umemoto, T., \& Kawabe, R. 2003, ApJ, 584, 357

Khanzadyan, T. 2003, Ph.D. Thesis

Lada, E. A., Strom, K. M., \& Myers, P. C. 1993, in Protostars and Planets III, 245

Lee, J.-S. 1986, Opt. Eng., 25, 636

Leous, J. A., Feigelson, E. D., Andre, P., \& Montmerle, T. 1991, ApJ, 379, 683

Loren, R. B. 1989a, ApJ, 338, 902

Loren, R. B. 1989b, ApJ, 338, 925

Loren, R. B., Wootten, A., \& Wilking, B. A. 1990, ApJ, 365, 269

Lucas, P. W., \& Roche, P. F. 1998, MNRAS, 299, 699

Luhman, K. L., \& Rieke, G. H. 1999, ApJ, 525, 440

Lynds, B. T. 1962, ApJS, 7, 1

Mezger, P. G., Sievers, A., Zylka, R., et al. 1992, A\&A, 265, 743

Montmerle, T., Koch-Miramond, L., Falgarone, E., \& Grindlay, J. E. 1983, ApJ, 269, 182

Motte, F., Andre, P., \& Neri, R. 1998, A\&A, 336, 150

Myers, P. C. \& Benson, P. J. 1983, Rev. Mex. Astron. Astrofis., 7, 238

Persson, S. E., Murphy, D. C., Krzeminski, W., Roth, M., \& Rieke, M. J. 1998, AJ, 116, 2475

Phelps, R. L., \& Barsony, M. 2004, AJ, 127, 420

Reipurth, B., Bally, J., Graham, J. A., Lane, A. P., \& Zealey, W. J. 1986, A\&A, 164, 51

Reipurth, B., Brand, J., Wouterloot, J. G. A., et al. 1991, European Southern Observatory Scientific Report, 11, 1

Rosen, A., \& Smith, M. D. 2004, MNRAS, 347, 1097

Sekimoto, Y., Tatematsu, K., Umemoto, T., et al. 1997, ApJ, 489, L63

Smith, M., Gredel, R., \& Khanzadyan, T., \& Stanke, T. 2004, A\&A, in preparation

Smith, M. D. 1995, A\&A, 296, 789

Smith, M. D., Suttner, G., \& Zinnecker, H. 1997, A\&A, 320, 325

Stanke, T. 2003, Ap\&SS, 287, 149

Stanke, T., Smith, M., Gredel, R., \& Khanzadyan, T. 2004, A\&A, submitted

Struve, O., \& Rudkjøbing, M. 1949, ApJ, 109, 92

Tachihara, K., Mizuno, A., \& Fukui, Y. 2000, ApJ, 528, 817

Tamura, M., Sato, S., Kaifu, N., Hough, J. H., \& Suzuki, H. 1990, ApJ, 350, 728

Terebey, S., Vogel, S. N., \& Myers, P. C. 1989, ApJ, 340, 472

Wilking, B. A., \& Lada, C. J. 1983, ApJ, 274, 698

Wilking, B. A., Lada, C. J., \& Young, E. T. 1989, ApJ, 340, 823

Wilking, B. A., Schwartz, R. D., Fanetti, T. M., \& Friel, E. D. 1997, PASP, 109, 549

Wu, J., Wang, M., Yang, J., Deng, L., \& Chen, J. 2002, AJ, 123, 1986

Young, E. T., Lada, C. J., \& Wilking, B. A. 1986, ApJ, 304, L45 